\title{
The value of dynamic contrast-enhanced $M R I$ in differentiating triple-negative breast cancer from other subtypes
}

\author{
Heba Azzam ${ }^{1,2^{*}} \mathbb{D}$, Rasha Kamal ${ }^{1}$, Hany El-Assaly ${ }^{1}$ and Lamiaa I. A. Metwally ${ }^{1}$
}

\begin{abstract}
Background: Breast cancer is a broad spectrum disease, including tumors showing different clinical, pathologic, molecular, and imaging features. Triple-negative breast cancer (TNBC) has an extra aggressive clinical course and poor prognosis being considered a diagnostic challenge to breast radiologists, yet it presented quite a lot of predictors on DCE-MRl; these could be valuable in identifying TNBC from other breast cancer subtypes.

In this study, we aimed at assessing the DCE-MRI features of triple-negative breast cancer in comparison to other subtypes of breast cancer.

Results: There was a significant difference between both groups regarding the internal enhancement pattern of mass lesions ( $P$ value 0.001), as well as intratumoral bright signal intensity on T2-weighted images ( $P$ value $<0.001)$. However, most of the breast cancer subtypes in this study showed malignant pattern kinetic curves type II and III showing no significant difference ( $P$ value 0.673).

Conclusion: TNBC presented several features with significant differences from other breast cancer molecular subtypes on DCE-MRI including the shape of the lesion and pattern of enhancement as well as high T2 signal intensity, thus improving the diagnosis of TNBC.
\end{abstract}

Keywords: Dynamic contrast-enhanced MRI, Triple-negative breast cancer

\section{Background}

Breast cancer is a broad spectrum disease, including tumors showing different clinical, pathologic, molecular, and imaging features. In addition to tumor size, pathological grade, and lymph node, the estrogen, progesterone, and HER2 receptor expression has important predictive value and guides the choice of the best treatment plan [1].

Based on DNA microarray techniques, gene expression profiling of breast cancer has classified breast cancer into five different molecular subtypes having recognized clinical, biologic, and therapeutic effects based on estrogen receptor (ER), progesterone receptor (PR), and HER-2 analysis, which are luminal A, luminal B, (HER $2 /$ neu)-overexpressing, normal breast-like tumor or unclassified group, and basal-like [2-6]. If the ER, PR, and

\footnotetext{
* Correspondence: heba.m.azzam@gmail.com

${ }^{1}$ Cairo University, Giza, Egypt

${ }^{2}$ Cairo, Egypt
}

SpringerOpen

(c) The Author(s). 2019 Open Access This article is distributed under the terms of the Creative Commons Attribution 4.0 International License (http://creativecommons.org/licenses/by/4.0/), which permits unrestricted use, distribution, and reproduction in any medium, provided you give appropriate credit to the original author(s) and the source, provide a link to the Creative Commons license, and indicate if changes were made.
HER-2/neu are not expressed in the breast cancer, it is called triple-negative breast cancer [7] (Table 1).

Triple-negative breast cancer (TNBC) has an extra aggressive clinical course and poor prognosis. They have an increased rate of local recurrence and metastatic disease, with a 5 -year survival of more or less $75 \%[8,9]$. The frequency of TNBC is high in younger, habitually premenopausal women $[1,10]$.

Imaging of TNBC is considered a diagnostic challenge to breast radiologists. On mammography and ultrasound, triple-negative cancer shows imprecise characteristics that can imitate lesions with benign features [11]. Patients with TNBC are commonly youthful with BRCA gene mutation, and they have dense breasts which additionally lowers mammographic sensitivity and specificity. The speedy progression of the TNBC which is not coupled with architectural distortions makes early detection of the disease exceptionally hard [12].

Breast MRI screening is beneficial for patients with high risk and those with occult breast carcinoma, which 
Table 1 Molecular classification of breast carcinoma [13]

\begin{tabular}{lllll}
\hline Feature & Luminal A & Luminal B & HER-2+ & Basal like \\
\hline ER/PR & Positive & Positive & Mostly negative & Negative \\
HER-2 & Negative & May be amplified & Amplified & Negative \\
Other markers' positivity & CK 8/18 & CK 8/18 & Androgen receptor & CK 5/6, EGFR \\
Proliferation rate (ki67 index) & Low & Intermediate & High & Very high \\
Recommended therapy & Hormonal & Hormonal and chemotherapy & Herceptin and chemotherapy & Chemotherapy \\
Prognosis & Favorable & Unfavorable & Poor & Worst \\
\hline
\end{tabular}

has a rather higher prevalence of TNBC $[14,15]$. MRI shows malignant characteristics that might not be recognized using mammography or ultrasound; consequently, it is considered of higher precision in detecting the local extent of disease in recently diagnosed breast cancer $[11,16]$. TNBC presented quite a lot of MRI predictors on DCE-MRI; these could be valuable in identifying TNBC from other breast cancer subtypes [10].

Therefore, the aim of this work is to assess the DCEMRI features of triple-negative breast cancer in comparison to other breast cancer subtypes and to validate its impact on management.

\section{Methods}

This study was a retrospective analysis, approved by the ethics committee during the period from October 2014 to March 2015, in which we analyzed the contrastenhanced MRI morphology descriptors and kinematic curve in 100 patients with triple-negative breast cancer as compared to another 100 patients (control group) of the same age group with different molecular subtypes.

\section{Patients}

It included 200 patients who were subjected to MRI scanning including pre-contrast and dynamic postcontrast sequences.

\section{Inclusion criteria}

Patients proved to have breast carcinoma of different molecular subtypes.

\section{Exclusion criteria}

- Cases that lacked pathological confirmation

- Cases proved to be benign

\section{Methods}

\section{MR imaging}

MRI was performed for the breasts using 1.5- $\mathrm{T}$ magnet scanners by two devices (Intera and Achieva, Philips medical system). All patients were examined in the prone position using a dedicated phased array breast coil with eight channels. Total study time ranged from 30 to $45 \mathrm{~min}$. No sedation was used.

\section{MRI imaging protocol}

A. Cases were examined first by pre-contrast sequences

Axial T1-weighted images, sagittal and axial T2weighted images, axial short time inversion recovery (STIR), and a pre-contrast fat-saturated T2-weighted pulse sequence. Pre-contrast images are obtained over a $512 \times 192$ matrix in the axial plane with a slice thickness of $4 \mathrm{~mm}$ without gap, flip angle $=90^{\circ}$ and FOV $=34-37$ $\mathrm{cm}$.

\section{B. Dynamic contrast-enhanced series}

A bolus of contrast (Magnevist (gadopentetatedimeglumine) $\quad 0.1 \mathrm{mmol} / \mathrm{kg}$ ) was injected manually intravenous.

\section{Post-processing and image analysis}

Post-processing image subtraction was obtained using the software subtraction function available on the work station to highlight the enhancing features in the image.

1. Quantitative analysis was done by using time/signal intensity curve.

A color overlay map was displayed, indicating regions of threshold enhancement.

2. The maximum intensity projection (MIP) (Fig.1a) was performed to demonstrate the distribution of disease in the breast in relation to the skin, nipple, chest wall, and large vessels.

\section{Image analysis}

Magnetic resonance images were reviewed by two radiologists in the same setting, and the final diagnosis was reached by their agreement (in consensus). The findings of dynamic contrast-enhanced MRI study were evaluated in accordance with the ACR BI-RADS MRI Lexicon, 2013, that includes two major categories of descriptors: morphology and enhancement kinetics. 

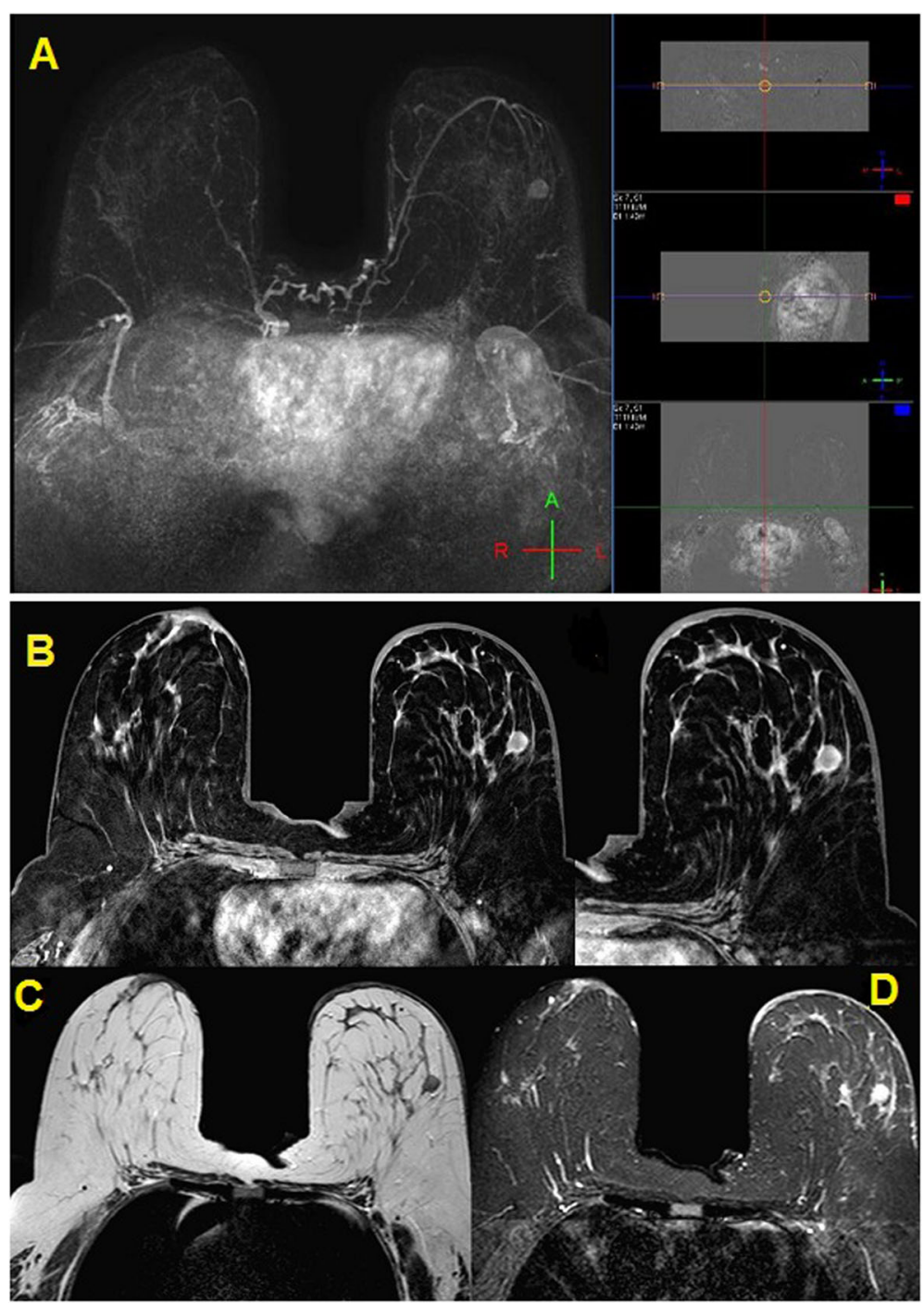

Fig. 1 MR images: a DCE-MRI MIP images, b post-contrast, c T2 WI, and $\mathbf{d}$ STIR WI. The images show left UOQ small not circumscribed (speculated) cystic mass lesion "arrow," eliciting intermediate T2 and high (bright) T2 STIR signal intensity. It shows rim enhancement on dynamic post-contrast images and markedly enlarged axillary lymph nodes (yellow arrow). The pathology revealed TN invasive ductal carcinoma

\section{A. Morphology descriptors}

Enhancing lesions were categorized as focus, mass, and non-mass enhancement.

1. Focus/foci: A focus is a breast lesion smaller than 5 mm (Fig. 2d).

2. Mass: A mass is a three-dimensional spaceoccupying lesion (Fig. 1).

(a) Multiplicity of masses

(b) Mass was characterized by the following:

Shape (oval, round, irregular), margin (circumscribed, not circumscribed) (Fig. 2), and internal enhancement characteristics (homogenous, heterogenous, rim enhancement (Fig. 3), dark internal septations).

3. Non-mass enhancement

(a) Distribution pattern

- Focal area

- Linear enhancement (Fig. 2d)

- Segmental enhancement 

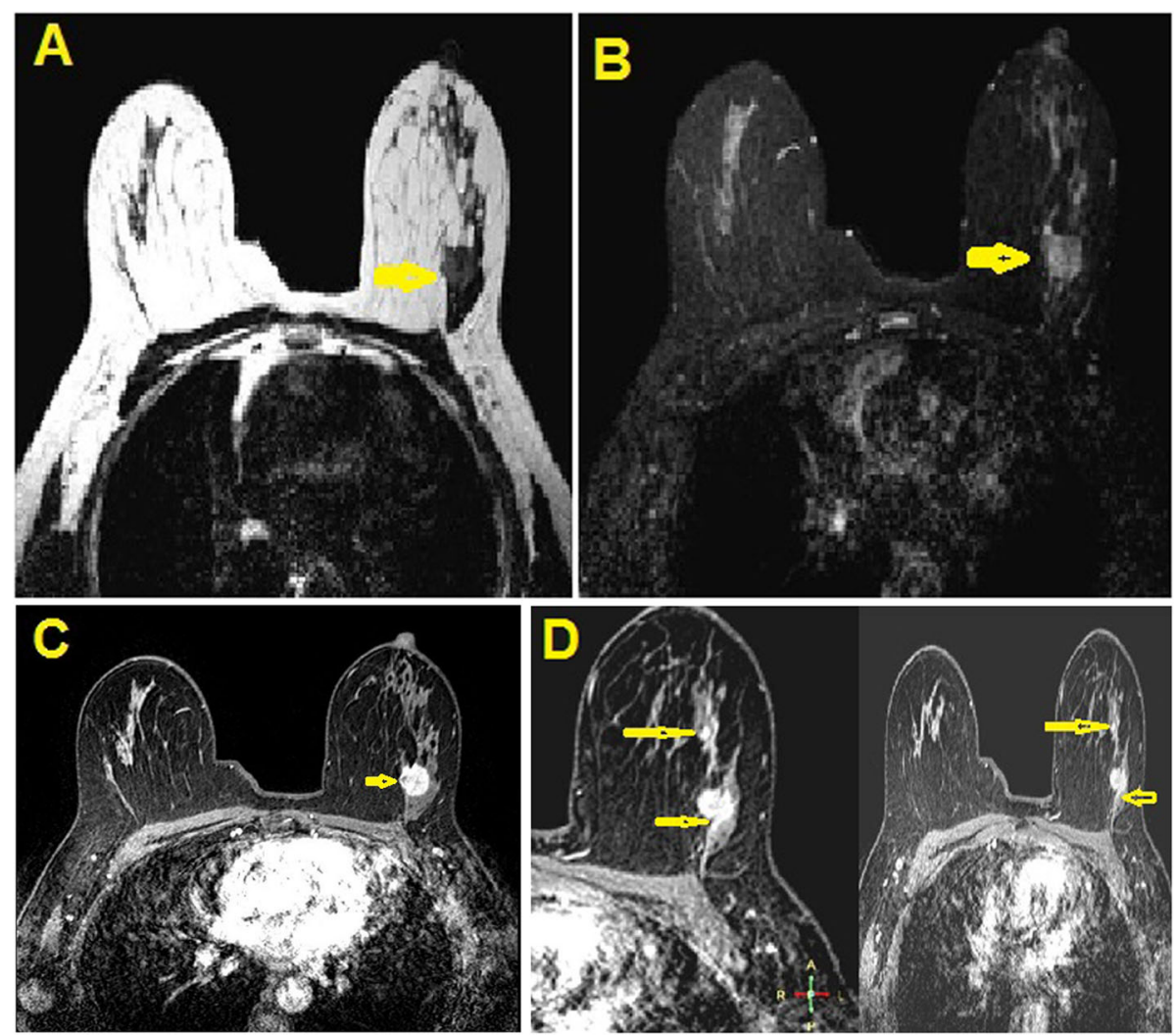

Fig. 2 MR images: a T2, b STIR, and c, d DCE-MRI post-contrast images. Left UOQ irregular non-circumscribed mass, eliciting intermediate-high T2 and STIR signal intensity "arrow." It shows heterogenous enhancement in dynamic post-contrast images "arrow." Left UOQ small linear non-mass lesion extending in front and behind the mass, eliciting bright T2 STIR signal intensity, and showing heterogeneous enhancement in dynamic post-contrast images "arrowheads." Left UOQ enhancing focus "arrow." The pathology revealed luminal B invasive ductal carcinoma

- Regional enhancement

- Diffuse enhancement

(b) Internal enhancement characteristics

- Homogenous

- Heterogeneous

- Clumped

- Clustered ring

\section{B. Associated features}

Associated findings with or without enhancement which include nipple retraction or inversion, skin retraction, thickening or invasion, lymphadenopathy, pectoral muscle invasion and chest wall invasion, architectural distortion, high signal intensity in ducts on unenhanced images, abnormal signal void, hematoma, edema, and cysts were reported.

Signs of spread of malignancy: On the MR images of the breast, spread to the axillary region, other lymph nodes, the chest wall muscles, the ribs, the adjacent pleura and lung, or the overlying skin was reported.

\section{Kinetic enhancement curve assessment}

Three enhancement patterns were identified on the basis of the time/signal intensity curve: types I, II, and III (Fig. 3g).

All patients were assigned a BI-RADS category [6] according to the ACR MRI BI-RADS lexicon.

\section{Statistical analysis}

The data was summarized using number and percentage for qualitative variables and mean and SD for normally distributed quantitative variables. Comparison between groups was done using the chi-square test for qualitative variables and the independent $T$ test for quantitative variables. $P$ value less than or equal to 0.05 was considered statistically significant, and 0.01 or less considered highly significant. 


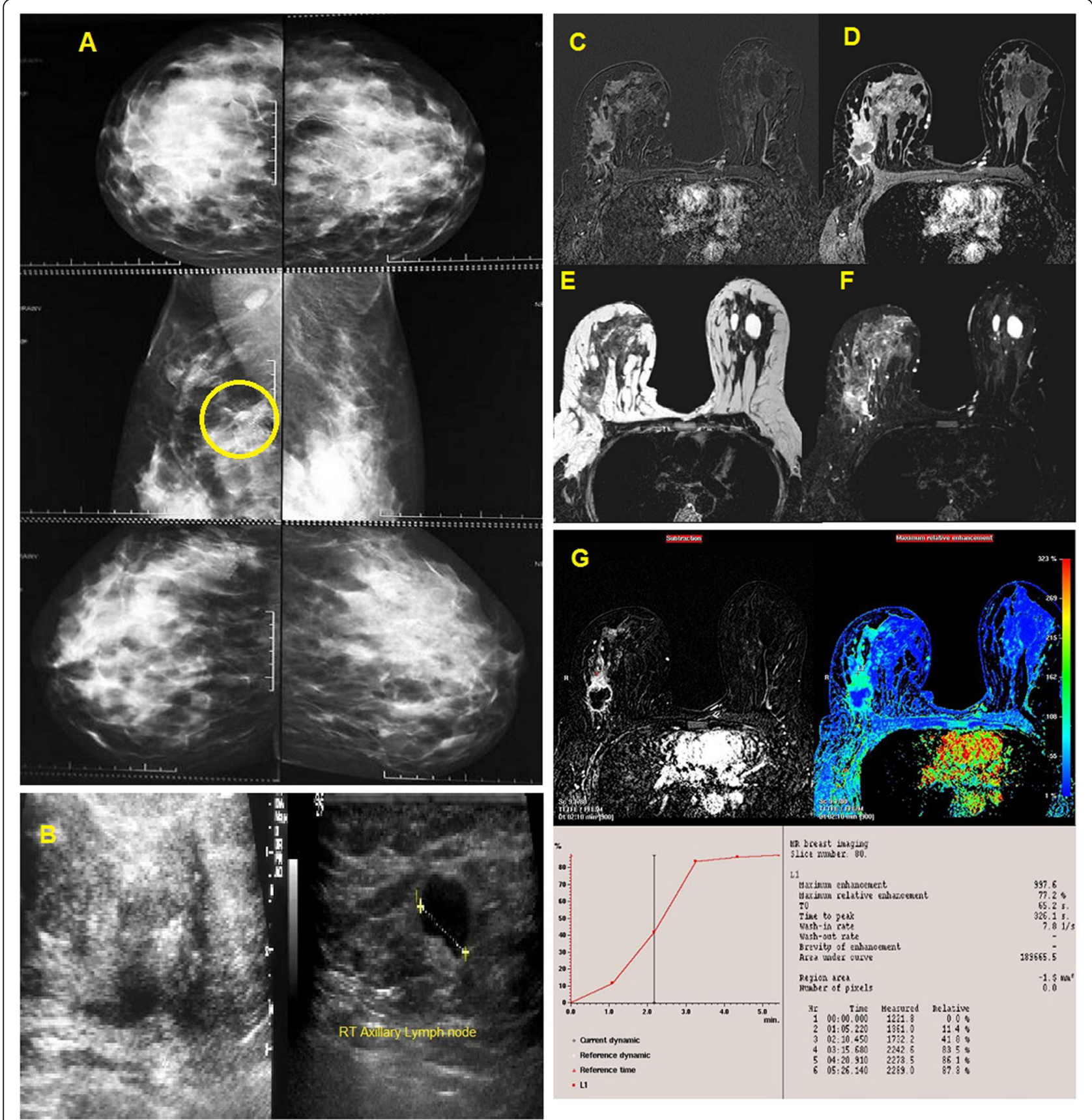

Fig. 3 a Mammography. b Ultrasound. c-f MRI images. c Subtraction. d Post-contrast. e T2 WI. f STIR WI. g Time/signal intensity curve. Mammography showing heterogeneous dense breast (ACR C). Right axillary tail pleomorphic clustered microcalcifications are seen (circle) with parenchymal distortion. Ultrasound showing ill-defined hypoehoic soft tissue lesion at the axillary tail of the right breast, with surrounding echogenic halo denoting desmoplastic reaction and parenchymal distortion with right pathologically enlarged axillary lymph nodes. MRI images show right UOQ large irregular speculated mass with surrounding area of architecture distortion, eliciting intermediate SI with areas of high T2 and STIR signal intensity, and displaying rim enhancement in dynamic post-contrast series. MRI time/signal intensity curve of right UOQ mass lesion shows delayed peak of contrast uptake and plateau (type II). The pathology revealed TN invasive ductal carcinoma.

\section{Results}

The study included 200 patients with 364 pathologically proved malignant breast lesions: 100 patients (with 172 lesions) TNBC and 100 patients (with 192 lesions) constituted the control group.
Age

Ages of TNBC patients ranged from 24 to 60 years (mean age 44.04 $\pm \mathrm{SD}$ ), while ages of control group patients ranged from 27 to 62 years (mean age 45.28 $\pm \mathrm{SD})$. 


\section{Clinical presentation}

Most of TNBC and control group patients presented with palpable mass lesions. No significant difference was presented between both groups.

\section{Histopathological diagnosis}

After revision of core biopsy reports, we found that TNBC 80/100 (80\%) cases were invasive ductal carcinoma, 16/100 (16\%) cases were lobular carcinoma, and 4/ $100(4 \%)$ cases were mucoid carcinoma, while in the control group, 84/100 (84\%) cases were IDC, 12/100 $(12 \%)$ cases were lobular carcinoma, and 4/100 (4\%) cases were mixed invasive and lobular carcinoma. All of them were positive for estrogen/progesterone receptors (luminal A and B).

\section{Type of lesion enhancement}

TNBC (172 lesions) distributed as 104/172 (60.5\%) showed mass enhancement, 52/172 (30.2\%) showed non-mass enhancement, and 16/172 (9.3\%) showed focus enhancement. In the control group (192 lesions), no significant difference was found as 108/192 (56.3\%) of the control lesions showed mass enhancement, 60/ 192 (31.3\%) of the control lesions showed non-mass enhancement, and 24/192 (12.5\%) of the control lesions showed focus lesions.

\section{Internal enhancement characteristics}

The predominant internal enhancement pattern of the TNBCs is rim enhancement. A highly significant difference was found between the two groups ( $P$ value 0.001$)$.

TNBC: $24 / 104$ (23.1\%) mass lesions showed homogenous enhancement, 32/104 (30.8\%) mass lesions showed heterogeneous enhancement, and 48/104 (46.2\%) mass lesions showed rim enhancement. In the control group, 76/108 (70.4\%) mass lesions showed homogenous enhancement, 24/108 (22.2\%) mass lesions showed heterogeneous enhancement, and 8/108 (7.4\%) mass lesions showed rim enhancement.

DCE-MRI features of TNBC compared to other subtypes of breast cancer (non-TNBC) are summarized in Table 2.

\section{Shape of the mass lesions}

TNBC: $40 / 104$ (38.5\%) were rounded or oval in shape and 64/104 (61.5\%) were irregular in shape.

Control group: 12/108 (11.1\%) were rounded or oval in shape and 96/108 (88.9\%) were irregular in shape.

\section{T2 signal intensity}

TNBC: 108/172 (62.8\%) lesions elicited high T2 signal intensity, 60/172 (34.9\%) lesions elicited low T2 signal intensity, and 4/172 (2.3\%) lesion was indistinct in T2weighted images. The increased T2 signal is due to the presence of necrotic areas which elicits high signal on T2 WI.

Control group: 32/192 (16.7\%) lesions elicited high T2 signal intensity, 152/192 (79.2\%) lesions elicited low T2 signal intensity, and 8/192 (4.2\%) lesions were indistinct in T2-weighted images.

\section{Discussion}

TNBC usually affects youthful females ( $<50$ years) [17], often presenting as palpable masses that might show benign characteristics on mammography and ultrasound, which might lead to faulty or delayed diagnosis [13].

Estrogen, progesterone, and HER2 receptor expression has important predictive value and guides the choice of the best treatment plan in addition to tumor size, pathological grade, and lymph node [1].

Better understanding of the DCE-MRI imaging features of TN cancers provides an imaging biomarker with clinical implications.

This study aimed to determine the dynamic contrastenhanced magnetic resonance imaging features of TN breast cancers in comparison to other breast cancer subtypes and to evaluate the DCE-MRI impact on the management of such cases.

Also, this study demonstrated certain MRI features with significant differences that are more frequent with triple-negative breast cancer compared with other breast cancer subtypes such as round shape mass lesion. Our results were consistent with some previous studies such as Sung et al. [1] who stated that $61 \%$ of TNBC lesions were lobulated, $25 \%$ irregular, and $14 \%$ round or oval with significant differences between the two groups ( $P$ value $<0.001)$, compared to other studies that reported most TNBC were rounded and/or oval masses [10, 18].

Other MRI features in which we found significant differences were mass with rim enhancement in postcontrast images and intratumoral bright signal intensity on T2-weighted images; this may be due to tumor necrosis compared to predominant homogenous internal enhancement in the other subtypes. Our results were consistent with many studies $[1,12,10,19-22]$ which affirmed that rim enhancement is the most beneficial MR characteristic for identifying TNBC and it is accompanied with more aggressive tumors [23].

Meanwhile, other DCE-MRI parameters showed no statistical differences between TN and non-TN breast cancer, which are the distribution of FGT and BPE, mass margin, non-mass enhancement distribution and enhancement pattern, lymph node status, and types of kinetic curves.

In this study, TNBC was diagnosed by DCE-MRI in all cases, and this coincides with previous studies such as Dogan et al. [20] who studied the characteristics of TNBC by mammography, ultrasound, and MR imaging; 
Table 2 DCE-MRI features of TNBC compared to other subtypes of breast cancer (non-TNBC)

\begin{tabular}{lll}
\hline DCE-MRI parameters & TNBC (\%) & Non-T \\
\hline FGT & & 0 \\
A/almost entirely fatty & 12 & 44 \\
B/scattered FGT & 56 & 48 \\
C/heterogenous FGT & 32 & 8 \\
D/extreme FGT & 0 &
\end{tabular}

BPE

Non-TNBC (\%) $\quad \frac{P \text { value }}{0.104}$

44

8

Minimal

40

Mild

36

Moderate

Type of lesion enhanced

Mass

NME

Mass associated with NME

Associated

Non-associated

Mass/shape

Oval

Round

Irregular

Mass/margin

Circumscribed

Non-circumscribed (irregular or spiculated)

Mass/internal enhancement

Homogenous

Heterogenous

Rim enhancement

NME/distribution

Focal

Linear

Segmental

Regional

Diffuse

NME/internal enhancement

Homogenous

Heterogenous

Clumped

T2 signal intensity

High

Low

Indistinct

T2 STIR signal intensity

High

Low

\section{2}

68

32


Table 2 DCE-MRI features of TNBC compared to other subtypes of breast cancer (non-TNBC) (Continued)

\begin{tabular}{lll}
\hline DCE-MRI parameters & TNBC (\%) & Non-TNBC (\%) \\
\hline Indistinct & 2.3 & 4.2 \\
Axillary lymph nodes & & 32 \\
Non-specific/not pathological & 28.0 & 68 \\
Pathological & 72.0 & \\
K. curve & & 16.2 \\
Type I: persistent & 16.7 & 29.7 \\
Type II: plateau & 38.9 & 54.1 \\
Type III: washout & 44.4 & 0.673 \\
\hline
\end{tabular}

TNBC were detected by MRI in all cases and in $91 \%$ and 93\% of cases by mammography and ultrasound respectively. Also, Schmadeka et al. [16] suggested that $18 \%$ of TNBC were not detected on mammography.

\section{Conclusion}

In conclusion, in this study, TNBC presented several features with significant differences from other breast cancer molecular subtypes on DCE-MRI including the shape of the lesion and pattern of enhancement as well as high $\mathrm{T} 2$ signal intensity, thus improving diagnosis of TNBC.

\section{Abbreviations}

ACR: American College of Radiology; BI-RADS: Breast Imaging-Reporting and Data System; BPE: Background parenchymal enhancement; BRCA gene: Breast cancer gene; DCE-MRI: Dynamic contrast-enhanced magnetic resonance imaging; ER: Estrogen receptor; FGT: Fibro-glandular tissue; HER2: Human epidermal growth factor receptor 2; IDC: Invasive ductal carcinoma; MIP: Maximum intensity projection; PCR: Pathologic complete response; PR: Progesterone receptor; TNBC: Triple-negative breast cancer; UOQ: Upper outer quadrant

\section{Acknowledgements}

Not applicable.

\section{Authors' contributions}

$H A, R K, H E$, and LM contributed equally to this work. HA and RK designed the research. HA and LM performed the research. HE and RK analyzed the data. HA and HE wrote the paper. All authors have read and approved the manuscript.

\section{Funding}

Not applicable (no funding received for this study)

\section{Availability of data and materials}

All the datasets used and analyzed during this study are available with the corresponding author on reasonable request.

\section{Ethics approval and consent to participate}

This study was approved by the research ethics committee of the Radiology Department of the Faculty of Medicine Cairo University on August 3, 2014, reference number of approval 768-2014.

All patients included in this study gave a written informed consent to participate in the research. If the patient was less than 16 years old, or unconscious at the time of the study, written informed consent was given by their parent or legal guardian.

\section{Consent for publication}

All patients included in this study gave a written informed consent to publish the data contained in this study. If the patient was less than 16 years old, or unconscious at the time of the study, written informed consent was given by their parent or legal guardian.

\section{Competing interests}

The authors declare that they have no competing interests.

Received: 6 September 2019 Accepted: 17 December 2019

Published online: 30 December 2019

\section{References}

1. Sung JS, Jochelson MS, Brennan S, Joo S, Wen YH, Moskowitz C, Zheng J, Dershaw DD, Morris EA (2013) MR imaging features of triple negative breast cancers. The Breast J. 19(6):643-649

2. Cetin I, Topcul M (2014) Triple negative breast cancer. Asian Pacific Journal of Cancer Prevention. 15(6):2427-2431

3. Boyle P (2012) Triple-negative breast cancer: epidemiological considerations and recommendations. Annals of Oncology 23(6):vi7-vi12

4. Kim JE, Ahn HJ, Ahn JH, Yoon DH, Kim S, Jung KH, Gong G, Kim M, Son BH, Ahn SH (2012) Impact of triple-negative breast cancer phenotype on prognosis in patients with stage I breast cancer. J Breast Cancer. 15(2):197202

5. Stevens KN, Vachon CM, Couch FJ (2013) Genetic susceptibility to triplenegative breast cancer. Cancer Res. 73(7):2025-2030

6. Yuan N, Meng M, Liu C, Feng L, Hou L, Ning Q, Xin G, Pei L, Gu S, Li X And Zhao X. (2014) Clinical characteristics and prognostic analysis of triplenegative breast cancer patients. Molecular And Clinical Oncology. 2(2):245251

7. Yadav BS, Sharma SC, Chanana P, Jhamb S (2014) Systemic treatment strategies for triple-negative breast cancer. World J Clin Oncol. 5(2):125-133

8. Krizmanich-Conniff KM, Paramagul C, Patterson SK, Helvie MA, Roubidoux MA, Myles JD, Jiang K, Sabel M (2012) Triple receptor-negative breast cancer: imaging and clinical characteristics. AJR Am J Roentgenol. 199(2): 458-464

9. Golden DI, Lipson JA, Telli ML, Ford JM, Rubin DL (2013) Dynamic contrastenhanced MRI-based biomarkers of therapeutic response in triple-negative breast cancer. J Am Med Inform Assoc. 20(6):1059-1066

10. Osman NM, Chalabi N, Abd Raboh NM (2014) Triple negative breast cancer: MRI features in comparison to other breast cancer subtypes with correlation to prognostic pathologic factors. Egypt J Radiol Nucl Med. 45(4):1309-1316

11. Youk JH, Son EJ, Chung J, Kim JA, Kim EK (2012) Triple-negative invasive breast cancer on dynamic contrast-enhanced and diffusion weighted MR imaging: comparison with other breast cancer subtypes. Euro Radiol. 22(8): 1724-1734

12. Boisserie-Lacroix M, MacGrogan G, Debled M, Ferron S, Asad-Syed M, McKelvie-Sebileau P, Mathoulin-Pélissier S, Brouste V, Hurtevent-Labrot G (2013) Triple-negative breast cancers: associations between imaging and pathological findings for triple-negative tumors compared with hormone receptor-positive/human epidermal growth factor receptor-2-negative breast cancers. The Oncologist. 18(7):802-811 
13. Voduc KD, Cheang MCU, Tyldesley S, Gelmon K, Nielsen TO, Kennecke H. Breast cancer subtypes and the risk of local and regional relapse. J Clin Oncol. 2010;28(10):1684-1691.

14. Dogan BE, Turnbull LW (2012) Imaging of triple-negative breast cancer. Annals of Oncology. 23(6):23-29

15. Li J, Han X (2014) Research and progress in magnetic resonance imaging of triple-negative breast cancer. Magnetic Resonance Imaging. 32(4):392-396

16. Atkins JJ, Appleton CM, Fisher CS, Gao F, Margenthaler JA (2013) Which imaging modality is superior for prediction of response to neoadjuvant chemotherapy in patients with triple negative breast cancer? Journal of Oncology 964863:7. https://doi.org/10.1155/2013/964863

17. Schmadeka R, Harmon BE, Singh M (2014) Triple-negative breast carcinoma current and emerging concepts. Am J Clin Pathol. 141(4):462-477

18. Ivanac G, Dominkovic MD, Brkljacic B, Zagreb HR, Orasje BA. Imaging features of triple negative breast carcinoma: mammography, ultrasound and MRI. European Society of Radiology. ECR 2014 / C-0482. DOl: https://doi.org/ 10.1594/ecr2014/C-0482.

19. Teifke A, Behr O, Schmidt M, Victor A, Vomweg TW, Thelen M, Lehr HA (2006) Dynamic MR imaging of breast lesions: correlation with microvessel distribution pattern and histologic characteristics of prognosis. Radiology. 239(2):351-360

20. Uematsu T, Kasami M, Yuen S (2009) Triple-negative breast cancer: correlation between MR imaging and pathological findings. Radiology. ORSNA 250(3):638-647

21. Dogan BE, Gonzalez-Angulo AM, Gilcrease M, Dryden MJ, Yang WT (2010) Multimodality imaging of triple receptor-negative tumors with mammography, ultrasound, and MRI. ARR Am J Roentgenol. 194(4):1160-1166

22. Costantini M, Belli P, Distefano D, Bufi E, Di Matteo M, Rinaldi P, Giuliani M, Petrone G, Magno S, Bonomo L (2012) Magnetic resonance imaging features in triple-negative breast cancer: comparison with luminal and HER2-overexpressing tumors. Clin Breast Cancer. 12(5):331-339

23. Lee SH, Cho N, Kim SJ, Cha JH, Cho KS, Ko ES, Moon WK (2008) Correlation between high resolution dynamic MRI features and prognostic factors in breast cancer. Korean J Radiol. 9(1):10-18

\section{Publisher's Note}

Springer Nature remains neutral with regard to jurisdictional claims in published maps and institutional affiliations.

\section{Submit your manuscript to a SpringerOpen ${ }^{\circ}$ journal and benefit from:}

- Convenient online submission

- Rigorous peer review

- Open access: articles freely available online

- High visibility within the field

- Retaining the copyright to your article

Submit your next manuscript at $\boldsymbol{\nabla}$ springeropen.com 